\title{
ON A LATTICE GENERALISATION OF THE LOGARITHM AND A DEFORMATION OF THE DEDEKIND ETA FUNCTION
}

\author{
LAURENT BÉTERMIN
}

(Received 14 September 2019; accepted 14 January 2020; first published online 20 February 2020)

\begin{abstract}
We consider a deformation $E_{L, \Lambda}^{(m)}(i t)$ of the Dedekind eta function depending on two $d$-dimensional simple lattices $(L, \Lambda)$ and two parameters $(m, t) \in(0, \infty)$, initially proposed by Terry Gannon. We show that the minimisers of the lattice theta function are the maximisers of $E_{L, \Lambda}^{(m)}(i t)$ in the space of lattices with fixed density. The proof is based on the study of a lattice generalisation of the logarithm, called the lattice logarithm, also defined by Terry Gannon. We also prove that the natural logarithm is characterised by a variational problem over a class of one-dimensional lattice logarithms.
\end{abstract}

2010 Mathematics subject classification: primary 33E20; secondary 11F20, 49K30.

Keywords and phrases: Dedekind eta function, theta function, lattice, optimisation.

\section{Introduction and setting}

Many mathematical models from physics are written in terms of special functions whose properties give fundamental information about the system (see, for example, [16]). For example, properties of the Jacobi theta function and the Dedekind eta function defined for $\mathfrak{J}(\tau)>0$ by

$$
\theta_{3}(\tau):=\sum_{k \in \mathbb{Z}} e^{-i \pi k^{2} \tau} \text { and } \eta(\tau):=q^{1 / 24} \prod_{n \in \mathbb{N}}\left(1-q^{n}\right), \quad q=e^{2 i \pi \tau},
$$

have been widely used to identify ground states of periodic systems (see, for example, $[4,10,13,19])$.

Generalisations and deformations of these special functions which arise in more complex physical systems are also of great interest. By a generalisation we mean that the summation appearing in the definition of the special function, which is usually on $\mathbb{Z}$ or a subset of $\mathbb{Z}$, is taken in a higher-dimensional lattice. By a deformation we mean that a parameter $m$, such as mass, is added to the initial function in such a way that the initial function is recovered in the limit $m \rightarrow 0$. While the lattice generalisation of

This research is supported by Villum Fonden via the QMATH Centre of Excellence (Grant No. 10059). (C) Cambridge University Press 2020. This is an Open Access article, distributed under the terms of the Creative Commons Attribution licence (http://creativecommons.org/licenses/by/4.0/), which permits unrestricted re-use, distribution, and reproduction in any medium, provided the original work is properly cited. 
special functions is the main topic of this paper, a typical example of deformation can be found in [1, Section 3.1] where some deformations of the Dedekind eta function (1.1) naturally arise in perturbative string theory when the cylinder diagrams that determine the static interactions between pairs of Dp-branes in the type IIB plane wave background are evaluated. One of these deformations, called $\eta^{(m)}$, was generalised by Gannon in [11], defining a new object called $E_{L, \Lambda}^{(m)}(i t)$ (see (1.4)). The aim of this paper is to optimise $(L, \Lambda) \mapsto E_{L, \Lambda}^{(m)}(i t)$ among a class of $d$-dimensional simple lattices, seen as parameters, for fixed $m, t>0$.

1.1. Simple lattices and energies. Before giving the precise definition of the deformation and generalisation of $\eta$, let us specify the spaces of lattices we are interested in. For any $d \geq 1$ and any $V>0$, we call $\mathcal{L}_{d}$ the set of all the simple lattices of the form

$$
L=\bigoplus_{i=1}^{d} \mathbb{Z} v_{i}, \quad \text { where }\left(v_{1}, \ldots, v_{d}\right) \text { is a basis of } \mathbb{R}^{d},
$$

and $\mathcal{L}_{d}^{\circ}(V)$ those with covolume $\left|\operatorname{det}\left(v_{1}, \ldots, v_{d}\right)\right|=V$, which is also the volume of the unit cell. For any $f$ such that the following series is absolutely convergent, it is natural to ask about the optimisation of

$$
L \mapsto E_{f}[L]:=\sum_{p \in L \backslash\{0\}} f\left(|p|^{2}\right)
$$

in $\mathcal{L}_{d}^{\circ}(V)$ for any fixed $V>0$. This problem has been studied in [15, 18] for the generalisation of the Jacobi theta function and the Riemann zeta function defined by

$$
\theta_{L}(\alpha):=\sum_{p \in L} e^{-\pi \alpha|p|^{2}} \quad(\alpha>0) \quad \text { and } \quad \zeta_{L}(s):=\sum_{p \in L \backslash\{0\}} \frac{1}{|p|^{s}} \quad(s>d),
$$

respectively called the lattice theta function and the Epstein zeta function (originally defined in $[9,12])$. Both functions are of interest because of their connection with other lattice 'energies' (see, for example, [2, 3, 5] and references therein). In particular, as explained in [2, Proposition 3.1], the energy $E_{f}$ of any function $f$ which is the Laplace transform of a nonnegative Borel measure $\mu$ and decays fast enough at infinity can be written as

$$
E_{f}[L]=\int_{0}^{\infty}\left(\theta_{L}(\alpha / \pi)-1\right) d \mu(\alpha)
$$

and it follows that an optimum $L_{d}$ for $L \mapsto \theta_{L}(\alpha)$ for all $\alpha>0$ is also an optimum for $E_{f}$. Such functions $f$ are called completely monotone and play an important role in optimal point configurations (see [7, 8]). Only three global optimality results have been shown for the lattice theta function.

- $\quad$ Montgomery [15]: if $d=2$, the triangular lattice

$$
\Lambda_{V}:=\sqrt{\frac{2 V}{\sqrt{3}}}\left[\mathbb{Z}(1,0) \oplus \mathbb{Z}\left(\frac{1}{2}, \frac{\sqrt{3}}{2}\right)\right]
$$

is the unique minimiser of $L \mapsto \theta_{L}(\alpha)$ in $\mathcal{L}_{2}^{\circ}(V)$ for all $V, \alpha>0$. 
- Cohn, Kumar, Miller, Radchenko and Viazovska [8]: if $d \in\{8,24\}, \mathrm{E}_{8}$ and the Leech lattice $\Lambda_{24}$ are the unique minimisers of $L \mapsto \theta_{L}(\alpha)$ in $\mathcal{L}_{d}^{\circ}(V)$ for all $V, \alpha>0$. More importantly, they are also the unique minimisers of this theta function among all periodic configurations of points (that is, where there are several points per unit cell).

The results we present in this paper are restricted to the simple lattice case. In order to have the most general results in this case, we call $\mathfrak{D}$ the set of dimensions $d$ such that $L \mapsto \theta_{L}(\alpha)$ has the same unique minimiser $L_{d}$ in $\mathcal{L}_{d}^{\circ}(V)$ for all $V, \alpha>0$. From the results just cited, we already know that $\{2,8,24\} \subset \mathbb{D} \neq \emptyset$. Furthermore, as explained in [20, page 117], $3 \notin \mathfrak{D} \neq \mathbb{N}$. From the above discussion, for all $d \in \mathfrak{D}$, the minimiser of the lattice theta function is the same for all the energies $E_{f}$ where $f$ is completely monotone.

1.2. Gannon's deformation of the Dedekind eta function. The goal of this paper is to use these results to find the maximal possible value of the deformed general eta function defined by Gannon in [11, Equation (3.5a)] as follows. Let $V>0, d \geq 1$, let $L, \Lambda \in \mathcal{L}_{d}^{\circ}(V)$ be two simple lattices and let $\mathcal{P}_{\Lambda}$ be the set of all vectors $v \in \Lambda$ such that the only scalar multiples $\lambda v \in \Lambda$ are integer multiples. We note that $v \in \mathcal{P}_{\Lambda}$ if and only if $-v \in \mathcal{P}_{\Lambda}$ and we write $\mathcal{P}_{\Lambda} / \pm$ for a subset of $\mathcal{P}_{\Lambda}$ with only one representative of each pair of vectors $\pm v \in \Lambda$. For any $m, t>0$, we define

$$
E_{L, \Lambda}^{(m)}(i t):=q^{-t^{(d-1) / 2} \Delta_{m}(L)} \prod_{w \in \mathcal{P}_{\Lambda} / \pm} \prod_{v \in L}\left(1-q^{|w|\left(m^{2}+|v|^{2}\right)}\right)^{t^{(d-1) / 2} / 2|w|}, \quad q=e^{-\pi t},
$$

where

$$
\Delta_{m}(L):=-\frac{1}{8 \pi|L|^{1 / 2}} \int_{0}^{\infty} s^{(d-1) / 2} e^{-\pi m^{2} / s}\left(\theta_{L^{*}}(s)-1\right) d s
$$

and $\theta_{L^{*}}$ is the lattice theta function defined by (1.3) for the dual lattice $L^{*}$ of $L$ defined by

$$
L^{*}:=\left\{y \in \mathbb{R}^{d}: y \cdot p \in \mathbb{Z} \text { for all } p \in L\right\} .
$$

For another formula which may look more familiar, see (3.1). This function $E_{L, \Lambda}^{(m)}$ can be viewed as a deformation of the Dedekind eta function $\eta$ defined by (1.1) in the following sense. We first remark that, for any $t>0$,

$$
E_{\mathbb{Z}, \mathbb{Z}}^{(m)}(i t)=: \eta^{(m)}(i t)
$$

where $(2 \pi m t)^{-1 / 2} \eta^{(m)}(i t) \rightarrow \eta(i t)$ as $m \rightarrow 0$. As explained before, the function $\eta^{(m)}(i t)$ appears in perturbative string theory [1]. We also notice that $\Delta_{m}$ is the lattice generalisation of the Casimir effect of a boson in a cylindrical worldsheet, the masslike parameter $m$ measuring how the background space-time (where the D-branes are located) is curved. 
1.3. Goal of our work. In order to explore the properties of this deformation of $\eta$, we ask the following question about extremal values for possible associated physical systems.

Question 1.1. What are the global maximisers of $(L, \Lambda) \mapsto E_{L, \Lambda}^{(m)}(i t)$ in $\mathcal{L}_{d}^{\circ}\left(V_{1}\right) \times \mathcal{L}_{d}^{\circ}\left(V_{2}\right)$ where $V_{1}, V_{2}>0$ and $m, t>0$ are fixed?

Studying $(L, \Lambda) \mapsto E_{L, \Lambda}^{(m)}($ it $)$ in $\mathcal{L}_{d}^{\circ}\left(V_{1}\right) \times \mathcal{L}_{d}^{\circ}\left(V_{2}\right)$ is an interesting mathematical question in itself which encourages us to investigate the maximality properties of other lattice energies, expanding the use of known variational techniques to new objects, like the lattice logarithm defined in the next subsection. Furthermore, even though $E_{L, \Lambda}^{(m)}(i t)$ does not appear yet in any model of (perturbative) string theory, we hope that our results will help mathematical physicists to optimise quantities like an analogue of the cylinder diagram in light-cone coordinates or the open-string one-loop diagram (see [1, Section 3]). We also believe that lattice sums of this type could be useful in number theory where such $q$-products appear frequently and are sometimes related to geometrical quantities, as $\eta$ is related to the height of the flat torus in dimension $d=2$ (see [17]). This problem is solved in Theorem 3.2, showing that for any $d \in \mathfrak{D}$, the minimisers of the lattice theta functions are the maximisers of $(L, \Lambda) \mapsto E_{L, \Lambda}^{(m)}(i t)$.

1.4. The lattice logarithm. As proposed by Gannon in the same paper (see [11, page 64]), we define the lattice generalisation of the logarithm as follows. This is connected to $E_{L, \Lambda}^{(m)}($ it) by (3.1).

Definition 1.2 (Lattice logarithm). For any $d \geq 1$, any $V>0$ and any simple lattice $L \in \mathcal{L}_{d}^{\circ}(V)$, we define the $L$-logarithm of any real number $x \in(0,1)$ by

$$
\log _{L}(x):=-\frac{1}{2} \sum_{p \in L \backslash\{0\}} \frac{(1-x)^{|p|}}{|p|} .
$$

Furthermore, if $d=1$ and $\left\{t_{n}\right\}_{n \in \mathbb{Z}}$ is an $N$-periodic sequence of real numbers for some $N \geq 1$, that is, such that $t_{n+N}-t_{n}=N$ for all $n \in \mathbb{Z}$, then the $\left\{t_{n}\right\}$-logarithm of $x \in(0,1)$ is defined by

$$
\log _{\left\{t_{n}\right\}}(x):=-\frac{1}{4 N} \sum_{i=1}^{N} \sum_{j \in \mathbb{Z} \backslash\{i\}} \frac{(1-x)^{\left|t_{j}-t_{i}\right|}}{\left|t_{j}-t_{i}\right|} .
$$

This $L$-logarithm is indeed a generalisation of the logarithm in the sense that $\log _{\mathbb{Z}}(x)=\log (x)$ for any $x \in(0,1)$. Gannon asked questions about the properties of this object.

1.5. Plan of the paper. In Section 2 , we study the maximisation of $L \mapsto \log _{L}(x)$ for given $x \in(0,1)$ as well as the problem of maximising a lattice energy (see (2.2)) of type $E_{f}$ defined as in (1.2), where $f$ depends on a lattice logarithm. The fact that the $L$-logarithm is summed over another lattice $\Lambda$ is the first example of this kind, when $L \neq \mathbb{Z}$. Finally, in Section 3, we prove our main result about the maximality of lattices $\left(V_{1}^{1 / d} L_{d}, V_{2}^{1 / d} L_{d}\right)$ for $E_{L, \Lambda}^{(m)}(i t)$ when $d \in \mathfrak{D}$ in $\mathcal{L}_{d}^{\circ}\left(V_{1}\right) \times \mathcal{L}_{d}^{\circ}\left(V_{2}\right)$. 


\section{Properties of the lattice logarithm}

We start by showing, in dimension $d=1$, that the natural logarithm is characterised by a maximality problem. This result allows us to construct functions, in a canonical way, that are smaller and close to $\log (x)$.

Theorem 2.1 (Characterisation of the logarithm). For any $x \in(0,1)$ and any $N$,

$$
\log (x)=\max _{\left\{t_{n}\right\}_{n \in \mathbb{Z}}}\left\{\log _{\left\{t_{n}\right\}}(x): t_{n+N}-t_{n}=N \text { for all } n \in \mathbb{Z}\right\}
$$

and this maximum is achieved if and only if $\left\{t_{n}\right\}=\mathbb{Z}+$ a for some $a \in \mathbb{R}$.

PRoof. For $x \in(0,1)$ and any $r>0$, let $\phi_{x}(r):=-2 \ell_{x}(r)$, where $\ell_{x}(r):=-(1-x)^{r} / 2 r$. We prove first that $\phi_{x}$ is strictly convex, that is, $\phi_{x}^{\prime \prime}(r)>0$ for any $r>0$. Indeed,

$$
\frac{(1-x)^{r}}{r}=\frac{e^{r \log (1-x)}}{r}=\int_{-\log (1-x)}^{\infty} e^{-t r} d t
$$

and it follows that $\phi_{x}^{\prime \prime}(r)=\int_{-\log (1-x)}^{\infty} t^{2} e^{-t r} d t>0$ on $(0, \infty)$.

By Ventevogel's optimality result [22, Theorem 1], for any $N \in \mathbb{N}$ and any $N$-periodic sequence $\left\{t_{n}\right\}$,

$$
\frac{1}{2 N} \sum_{i=1}^{N} \sum_{j \in \mathbb{Z} \backslash\{i\}} \phi_{x}\left(\left|t_{j}-t_{i}\right|\right) \geq \sum_{k=1}^{\infty} \phi_{x}(k) .
$$

Thus,

$$
\log _{\left\{t_{n}\right\}}(x) \leq \sum_{k=1}^{\infty} \ell_{x}(k)=-\frac{1}{2} \sum_{k=1}^{\infty} \frac{(1-x)^{k}}{k}=\log _{\mathbb{Z}}(x)=\log (x),
$$

and our result is proved because $\left\{t_{n}\right\}=\mathbb{Z}+a$ are the only sequences satisfying the equality case in Jensen's inequality.

Remark 2.2. One might be tempted to think that this result is true for any kind of lattice generalisation of a classical function, but one would be wrong. Indeed, we can define a $\left\{t_{n}\right\}$-exponential for $x>0$ by

$$
\exp _{\left\{t_{n}\right\}}(x):=\frac{1}{2 N} \sum_{i=1}^{N} \sum_{j \in \mathbb{Z} \backslash\{i\}} \frac{x^{\left|t_{j}-t_{i}\right|}}{\Gamma\left(\left|t_{j}-t_{i}\right|+1\right)},
$$

and ask the same question about the global optimality of $\left\{t_{n}\right\}=\mathbb{Z}+a$. It turns out that the function $\psi_{x}(r)=x^{r} / \Gamma(r+1)$ is not convex for some values of the parameter, for example $x=e$, so our method cannot be used. Furthermore, for $x=e$, the function is not even decreasing on $(0, \infty)$, which makes it a bad candidate for the optimality of the equidistant configuration.

In higher dimensions, $d \geq 2$, we restrict our study to the simple lattice case and we have the following result connecting the minimisers of the lattice theta function and that of $L \mapsto \log _{L}(x)$ for any given real number $x \in(0,1)$. 
Theorem 2.3 (Maximiser of the $L$-logarithm). Let $d \in \mathfrak{D}, x \in(0,1)$ and $V>0$. Then the unique minimiser $V^{1 / d} L_{d}$ of the lattice theta function is the unique maximiser of $L \mapsto \log _{L}(x)$ in $\mathcal{L}_{d}^{\circ}(V)$.

PRoof. It is sufficient to show that $\phi_{x}(r):=(1-x)^{\sqrt{r}} / \sqrt{r}$ is a completely monotone function and to use [2, Proposition 3.1] already mentioned in the introduction to complete the proof. Writing $\phi_{x}(r)=e^{a \sqrt{r}} / \sqrt{r}$ and remarking that $a:=\log (1-x)<0$ shows that $\phi_{x}$ is completely monotone as a product of completely monotone functions (see [14]).

Since $\log _{L}$ is a function by itself on $(0,1)$, we can use it to define a new lattice sum where the $L$-logarithm is an interacting potential. The problem of finding optimal point configurations for logarithmic interaction is indeed of great interest in dimension $d=2$ [21] (where $-\log$ is the Coulomb potential) and on the $(d-1)$-dimensional sphere [6]. We therefore obtain an energy depending on two simple lattices that we can maximise as follows.

Theorem 2.4 (The $L$-logarithm viewed as interacting potential). Let $d \in \mathfrak{D}$ and $V_{1}$, $V_{2}>0$. For any simple lattices $L \in \mathcal{L}_{d}^{\circ}\left(V_{1}\right)$ and $\Lambda \in \mathcal{L}_{d}^{\circ}\left(V_{2}\right)$ and for any function $f$ such that $f^{\prime}$ is completely monotone, we define

$$
\mathcal{E}_{f}[L, \Lambda]:=\sum_{q \in \Lambda} \log _{L}\left(1-e^{-f\left(|q|^{2}\right)}\right)
$$

Then, for any fixed $L$ and $f, V_{2}^{1 / d} L_{d}$ is the unique maximiser of $\Lambda \mapsto \mathcal{E}_{f}[L, \Lambda]$ in $\mathcal{L}_{d}^{\circ}\left(V_{2}\right)$. Furthermore, $\left(V_{1}^{1 / d} L_{d}, V_{2}^{1 / d} L_{d}\right)$ is the unique pair of maximisers of $\mathcal{E}_{f}$ in $\mathcal{L}_{d}^{\circ}\left(V_{1}\right) \times \mathcal{L}_{d}^{\circ}\left(V_{2}\right)$.

Proof. By absolute convergence,

$$
\mathcal{E}_{f}[L, \Lambda]=-\frac{1}{2} \sum_{q \in \Lambda} \sum_{p \in L \backslash\{0\}} \frac{e^{-|p| f\left(|q|^{2}\right)}}{|p|}=-\frac{1}{2} \sum_{p \in L \backslash\{0\}} \frac{1}{|p|}\left(\sum_{q \in \Lambda} e^{-|p| f\left(|q|^{2}\right)}\right)
$$

and it is clear that the energy is well defined. Since $f^{\prime}$ is a completely monotone function and $r \mapsto e^{-|p| r}$ is also completely monotone for any $p \in L \backslash\{0\}$, it follows from [14, Theorem 2] that $r \mapsto e^{-|p| f(r)}$ is completely monotone. Therefore, by [2, Proposition 3.1], $V_{2}^{1 / d} L_{d}$ is the unique minimiser of $\Lambda \mapsto \sum_{q \in \Lambda} e^{-|p| f\left(|q|^{2}\right)}$ in $\mathcal{L}_{d}^{\circ}\left(V_{2}\right)$ and the first part of the theorem is proved. The second part follows from the first part by using (2.3) and Theorem 2.3.

REMARK 2.5. As already mentioned in the introduction, it is, as far as we know, the first time that an energy with an interaction potential depending on a lattice has been studied. It might be interesting to consider a lattice generalisation of the lattice theta function, using the $L$-exponential defined by (2.1), and see if one can again derive optimality results of this kind. The fact that the associated potential $\psi_{x}$ defined in Remark 2.2 is not convex might be a problem but also an interesting direction to explore. 


\section{Maximisation of $E_{L, \Lambda}^{(m)}(i t)$ among simple lattices}

It is straightforward to obtain the following formula that connects $E_{L, \Lambda}^{(m)}(i t)$ to the lattice logarithm.

Lemma 3.1 [11]. For any $t, m>0$ and any simple lattices $L, \Lambda \in \mathcal{L}_{d}$,

$$
\begin{aligned}
\log E_{L, \Lambda}^{(m)}(i t)=- & \frac{t^{(d+1) / 2}}{8|L|^{1 / 2}} \int_{0}^{\infty} s^{(d-1) / 2} e^{-\pi m^{2} / s}\left(\theta_{L^{*}}(s)-1\right) d s \\
& +t^{(d-1) / 2} \sum_{p \in L} \log _{\Lambda}\left(1-q^{m^{2}+|p|^{2}}\right) .
\end{aligned}
$$

Therefore, we can easily derive the maximum of $(L, \Lambda) \mapsto E_{L, \Lambda}^{(m)}(i t)$ among simple lattices.

Theorem 3.2 (Maximiser of $E_{L, \Lambda}^{(m)}(i t)$ ). Let $d \in \mathfrak{D}, t, m>0$ and $V_{1}, V_{2}>0$. Then $\left(V_{1}^{1 / d} L_{d}, V_{2}^{1 / d} L_{d}\right)$ is the unique maximiser of $(L, \Lambda) \mapsto E_{L, \Lambda}^{(m)}($ it $)$ in $\mathcal{L}_{d}^{\circ}\left(V_{1}\right) \times \mathcal{L}_{d}^{\circ}\left(V_{2}\right)$.

Proof. We use formula (3.1). Since $d \in \mathfrak{D}$, it follows that $s^{(d-1) / 2} e^{-\pi m^{2} / s}>0$ for any $s>0$ and $L_{d}=L_{d}^{*}$. As a simple consequence of the Poisson summation formula,

$$
L \mapsto \int_{0}^{\infty} s^{d-1 / 2} e^{-\pi m^{2} / s}\left(\theta_{L^{*}}(s)-1\right) d s
$$

is minimised by $V_{1}^{1 / d} L_{d}$ in $\mathcal{L}_{d}^{\circ}\left(V_{1}\right)$. Therefore the first term is maximised by $V_{1}^{1 / d} L_{d}$. For the second term, we use Theorem 2.4 with $f(r)=\pi t\left(m^{2}+r\right)$ and we remark that $f^{\prime}(r)=\pi t$ is a completely monotone function. This implies that the second term is maximised by $\left(V_{1}^{1 / d} L_{d}, V_{2}^{1 / d} L_{d}\right)$, which concludes the proof.

\section{References}

[1] O. Bergman, M. R. Gaberdiel and M. B. Green, 'D-brane interactions in type IIB plane-wave background', J. High Energy Phys. 2003 (2003), 002.

[2] L. Bétermin, 'Two-dimensional theta functions and crystallization among Bravais lattices', SIAM J. Math. Anal. 48(5) (2016), 3236-3269.

[3] L. Bétermin, 'Minimizing lattice structures for Morse potential energy in two and three dimensions', J. Math. Phys. 60(10) (2019), 102901.

[4] L. Bétermin and H. Knüpfer, 'On Born's conjecture about optimal distribution of charges for an infinite ionic crystal’, J. Nonlinear Sci. 28(5) (2018), 1629-1656.

[5] L. Bétermin and H. Knüpfer, 'Optimal lattice configurations for interacting spatially extended particles', Lett. Math. Phys. 108(10) (2018), 2213-2228.

[6] J. S. Brauchart and P. Grabner, 'Distributing many points on spheres: minimal energy and designs', J. Complexity 31 (2015), 293-326.

[7] H. Cohn and A. Kumar, 'Universally optimal distribution of points on spheres', J. Amer. Math. Soc. 20(1) (2007), 99-148.

[8] H. Cohn, A. Kumar, S. D. Miller, D. Radchenko and M. Viazovska, 'Universal optimality of the E8 and Leech lattices and interpolation formulas', Preprint, 2019, arXiv:1902:05438.

[9] P. Epstein, 'Zur Theorie allgemeiner Zetafunctionen', Math. Ann. 56(4) (1903), 615-644. 
[10] M. Faulhuber, 'Extremal determinants of Laplace-Beltrami operators for rectangular tori', Preprint, 2017, arXiv:1709.06006.

[11] T. Gannon, 'Variations on Dedekind's eta', in: Symmetry in Physics: in Memory of Robert T. Sharp, CRM Proceedings and Lecture Notes, 34 (American Mathematical Society, Providence, RI, 2005), 55-66.

[12] A. Krazer and E. Prym, Neue Grundlagen einer Theorie der allgemeinen Theta-funktionen (Teubner, Leipzig, 1893).

[13] S. Luo, X. Ren and J. Wei, 'Non-hexagonal lattices from a two species interacting system', Preprint, 2019, arXiv:1902.09611.

[14] K. S. Miller and S. G. Samko, 'Completely monotonic functions', Integral Transforms Spec. Funct. 12 (2001), 389-402.

[15] H. L. Montgomery, 'Minimal theta functions', Glasg. Math. J. 30(1) (1988), 75-85.

[16] A. F. Nikiforov and V. B. Uvarov, Special Functions in Mathematical Physics. A Unified Introduction with Applications (Birkhäuser, Basel, 1988).

[17] B. Osgood, R. Phillips and P. Sarnak, 'Extremals of determinants of Laplacians', J. Funct. Anal. 80 (1988), 148-211.

[18] R. A. Rankin, 'A minimum problem for the Epstein zeta-function', Proc. Glasg. Math. Assoc. 1(4) (1953), 149-158.

[19] E. Sandier and S. Serfaty, 'From the Ginzburg-Landau model to vortex lattice problems', Comm. Math. Phys. 313(3) (2012), 635-743.

[20] P. Sarnak and A. Strömbergsson, 'Minima of Epstein's zeta function and heights of flat tori', Invent. Math. 165 (2006), 115-151.

[21] S. Serfaty, 'Systems of points with Coulomb interactions', EMS Newsl. 12 (2018), 16-21.

[22] W. J. Ventevogel, 'On the configuration of systems of interacting particle with minimum potential energy per particle', Physica A 92(3-4) (1978), 343-361.

LAURENT BÉTERMIN, Faculty of Mathematics, University of Vienna, Oskar-Morgenstern-Platz 1, 1090 Vienna, Austria

e-mail: laurent.betermin@univie.ac.at 